\title{
Towards reconciling the holographic and lattice descriptions of radially excited hadrons
}

\author{
S. S. Afonin ${ }^{\mathrm{a}}$ \\ Saint Petersburg State University, 7/9 Universitetskaya nab., St. Petersburg 199034, Russia
}

Received: 22 April 2020 / Accepted: 30 July 2020 / Published online: 11 August 2020

(C) The Author(s) 2020

\begin{abstract}
Within the framework of AdS/QCD models, the spectra of radially excited hadrons are identified with towers of Kaluza-Klein (KK) states in a putative dual theory. The infinite number of KK states is indispensable to provide correct high energy asymptotics of correlation functions in QCD. It is known, however, that the KK modes of dual theory must be qualitatively different from real hadrons. And what is more important, the radially excited states appear in lattice calculations not as "excitations" of some ground state, but rather as independent states coupled to higher dimensional QCD operators - the larger is a basis of interpolating operators, the larger set of states can be resolved. A question arises whether is it possible to reconcile the holographic and lattice descriptions of radially excited hadrons? We propose a new phenomenological "consistency test" for bottom-up holographic models: If the KK spectrum of massive 5D fields dual to higher dimensional operators in QCD coincides with the conventional radial KK spectrum, then the holographic KK states can be identified with real excited mesons in the large- $N_{c}$ limit of QCD. We demonstrate that the Soft Wall holographic model passes this test while the Hard Wall model does not.
\end{abstract}

\section{Introduction}

The confinement in QCD is known to lead to a rich spectrum of excited hadrons. A complete theoretical understanding of this spectrum is still missing despite of many fruitful ideas and models put forward in the last half of century. The problem becomes especially acute in the case of hadrons composed from light quarks where one observes plenty of higher spin and radial excitations. The corresponding hadron resonances usually have large decay widths and this causes many difficulties not only with experimental extraction of their characteristics but also with a clear theoretical identifi-

\footnotetext{
a e-mail: s.afonin@spbu.ru (corresponding author)
}

cation of the given objects. In this situation, it is very useful to consider a limit where many hadron resonances become well-defined particles while the underlying theory remains qualitatively similar. Quite remarkably, such a limit indeed exists in QCD - the large- $N_{c}$ (called also planar) limit [1], in which the quark-antiquark states become stable particles: Both their strong decay width and corrections to masses are suppressed by $1 / N_{c}$. Baryons represent heavy objects in this limit [2] (their mass behaves as $\mathcal{O}\left(N_{c}\right)$ while the mass of quark-antiquark mesons scales as $\mathcal{O}(1)$ ) and will be of no interest for us. Matching the obtained meson theory to the perturbative QCD at small 't Hooft constant $\lambda \doteq g^{2} N_{c}$ shows that the theory at small $\lambda$ must contain an infinite number of states for each quantum number [2] - the "radial" excitations in the language of non-relativistic potential models.

It has long been suspected that planar QCD is equivalent to some string theory. This idea got a new push with the advent of AdS/CFT (or more generically holographic) correspondence [3-6]. The use of holographic conjectures has now penetrated many branches of theoretical physics including the real QCD (this branch of research is referred to as holographic QCD or AdS/QCD). Within the holographic QCD, the infinite number of mesons with identical quantum numbers expected in the large- $N_{c}$ limit is modelled by an infinite tower of Kaluza-Klein (KK) excitations. The most popular phenomenological models of this sort are the Hard Wall (HW) [7,8] and Soft Wall (SW) [9] holographic models. They have enjoyed an unexpected phenomenological success - the HW model was particularly successful in description of physics related to the chiral symmetry breaking in QCD and of hadron form factors while the SW one turned out to be very convenient in description of phenomenology of stringlike linear Regge trajectories.

The modeling of radially excited mesons by KK modes looks, however, too simplistic - the former are highly complicated dynamical objects in QCD while the latter are rather simple states arising from extra dimension. The dramatic dif- 
ference between the KK-like and QCD-like states is discussed in detail in Ref. [10]. The main point consists in observation that the KK modes are deeply bound states sensitive to short distance interactions and at collisions producing events with mostly spherical shapes while mesons are extended states sensitive to large distance interactions and producing characteristic jets. The underlying reason is that the latter are defined at small 't Hooft coupling $\lambda$ while the former at large $\lambda$ where the existence of holographic duality can be motivated. The theories at small and large $\lambda$ turn out to be qualitatively different. In Sect. 5, we will add to this discussion by indicating rather hard difficulties with a correct definition of propagating $\mathrm{KK}$ modes (except the lightest one) because the bulk space in the holographic duality is not exactly the AdS one.

We see thus that the straightforward identification of KK states with the real large- $N_{c}$ mesons - what is always implied in AdS/QCD models - has problems. To address these problems, it is instructive to recall how the radially excited states are described from first principles. Within the lattice calculations, these states appear not as "excitations" of some ground state, but rather as independent states arising from Higher Dimensional Operators (HDO) in QCD in the sense that the larger basis of interpolating operators is included into simulations, the larger number of states can be resolved [11]. For a consistent holographic description of real mesons, it looks therefore natural to introduce massive fields dual to HDO in QCD. These fields will have their own discrete spectra of normalizable modes. Our idea is the following: If the KK spectrum of each of new heavier fields coincide with the original KK spectrum since some mode of original KK spectrum, we can draw a one-to-one correspondence between each higher KK mode and a set of QCD operators leading to the mass of this mode. We get then a self-consistent physical picture - on the one hand, the infinite tower of formal KK states provides a correct high energy asymptotics for hadronic correlation functions (the importance of this requirement for internal consistency of the model was recently emphasized in Refs. [12-14]), on the other hand, each KK state in this tower can be traded for a state interpolated by a definite set of QCD operators and thus interpreted as a real excited meson in the large- $N_{c}$ limit.

We will demonstrate how the outlined scheme works in the SW model. In the case of HW model, however, the spectra of KK states and states emerging from HDO are different. We interpret this as another one argument (in addition to nonRegge like behavior and absence of power-like corrections in the OPE) that the KK states of HW model cannot be identified with real hadrons. It is interesting to observe, however, that the spectrum of ground states related with HDO rises much slower with excitation number than the spectrum of radial KK states and qualitatively approaches the rise of experimental radial trajectories.
Finally it should be mentioned that inclusion of new 5D fields dual to higher dimensional operators in $4 \mathrm{D}$ gauge theory improves the consistency of AdS/QCD models from the viewpoint of general principles of gauge/gravity duality. We remind the reader that within the original AdS/CFT correspondence conjectured in the type IIB superstring theory, for description of 4D gauge theory one needs not only low-energy supergravity on $\mathrm{AdS}_{5}$ but also the whole infinite tower of massive KK states on $\mathrm{AdS}_{5} \times \mathrm{S}_{5}$ which appear after the KK-compactification on 5D sphere [5]. These 5D KK states are "enumerated" by scaling dimensions of higherdimensional operators in $4 \mathrm{D}$ gauge theory. The given point is of crucial importance in establishing a complete mapping of physical degrees of freedom between the two dual theories.

The paper is organized as follows. In Sect. 2, we begin with a very brief review on the HDO in QCD. Then we apply our idea to the SW and HW holographic models in Sects. 3 and 4 correspondingly. Further discussions are contained in Sect. 5 and we conclude in Sect. 6.

\section{Higher dimensional QCD operators}

The general idea that radially excited hadrons should arise from coupling to HDO in QCD is rather old and has appeared from time to time in the literature. It was used, for instance, for construction of some extended low-energy quark models [15-19]. And what is the most important, this idea is explicitly realized in the lattice calculations of radial excitations [11].

Consider the following two basic quark currents,

$$
\begin{aligned}
& V_{\mu}=\bar{q} \gamma_{\mu} q=\bar{q}_{\mathrm{L}} \gamma_{\mu} q_{\mathrm{L}}+\bar{q}_{\mathrm{R}} \gamma_{\mu} q_{\mathrm{R}}, \\
& S=\bar{q} q=\bar{q}_{\mathrm{L}} q_{\mathrm{R}}+\bar{q}_{\mathrm{R}} q_{\mathrm{L}} .
\end{aligned}
$$

Here the Dirac spinor $q=q_{\mathrm{L}}+q_{\mathrm{R}}$ stays for $u$ or $d$ quark fields and $q_{\mathrm{L}, \mathrm{R}}=\frac{1 \mp \gamma_{5}}{2} q$. The isospin and $\gamma_{5}$ matrices can be also inserted in (1) and (2) but this is not essential for our discussions and will be dropped (but implicitly assumed where necessary). What is essential is the different chiral structure of twist-2 vector current (1) and twist- 3 scalar current (2) - they transform differently under $S U_{\mathrm{L}}(2) \times S U_{\mathrm{R}}(2)$ chiral transformations. The chiral transformation properties of QCD currents were discussed in detail in many papers and we refer to the relevant literature (see, e.g., [20-23]).

The twist-2 vector current (1) has been traditionally used for interpolation of $\rho$ and $\omega$ mesons in QCD sum rules, lattice QCD and low-energy effective field theories. Consider now the lightest $\rho_{3}$ excitation - the resonance $\rho_{3}(1690)$ [24]. The leading twist spin-3 quark operator can be easily constructed by insertion of covariant derivatives (the appropriate symmetrization is implied),

$V_{\mu_{1} \mu_{2} \mu_{3}}=\bar{q} \gamma_{\mu_{1}} D_{\mu_{2}} D_{\mu_{3}} q$. 
The tensor current (3) is not conserved (as the scalar one (2) and many others) but the experience of spectral QCD sum rules shows that the conservation is of no importance for finding the relevant pole [25-27]. The interpolating operator (3) repeats the chiral properties of (1). We can contract the last two Lorentz indices in (3) and get the twist-4 vector current

$V_{\mu}^{\prime}=\bar{q} \gamma_{\mu} D^{2} q$.

It is natural to expect that the current (4) couples to a spin-1 state lying in a mass range close to $\rho_{3}(1690)$. Such a state does exists - the resonance $\rho(1700)$ [24]. There are many other cases of approximate degeneracy between spin and radial excitations and this gives rise to a remarkable general picture of degeneracies in the experimental spectrum of light non-strange mesons up to $2.5 \mathrm{GeV}$ [23,28-33]. We obtain thus a kind of "experimental" confirmation for the conjecture that the radially excited states couple to HDO in QCD.

On the other hand, a vector interpolating current can be constructed also by insertion of covariant derivative to the scalar current (2),

$\tilde{V}_{\mu}=\bar{q} D_{\mu} q=\bar{q}_{\mathrm{L}} D_{\mu} q_{\mathrm{R}}+\bar{q}_{\mathrm{R}} D_{\mu} q_{\mathrm{L}}$.

This operator inherits the chiral properties of the current (2) and its twist. In addition, the currents (1) and (5) look different from the point of view of Lorentz group since (5) can be represented on shell as [23]

$\bar{q} D_{\mu} q \propto-\partial^{\nu} H_{\mu \nu}, \quad H_{\mu \nu}=\bar{q} \sigma_{\mu \nu} q$,

where $\sigma_{\mu \nu}=\left(\gamma_{\mu} \gamma_{\nu}-\gamma_{\nu} \gamma_{\mu}\right) / 2 i$. The antisymmetric tensor current $H_{\mu \nu}$ transforms as $(1,0)+(0,1)$ while (1) has the Lorentz structure $\left(\frac{1}{2}, \frac{1}{2}\right)$ [19-23]. One can notice that the vector current $\partial^{v} H_{\mu \nu}$ is trivially conserved but this conservation is topological, i.e. of different nature than the conservation of Noether current (1). What $\rho$-meson does the operator (5) interpolate? Since it has one covariant derivative, one can expect the corresponding state to lie between $\rho(770)$ and $\rho(1700)$. There exists one well-established $\rho$-meson in this range - the resonance $\rho(1450)$ [24]. One may expect also that the experimental study of this resonance is more difficult because its production should be suppressed in $e^{+} e^{-}$annihilation. The Particle Data [24] indeed makes caution that $\rho(1450)$ is the name for a broad resonance region rather than a definite resonance. The first suggestion to associate (6) with $\rho(1450)$ appeared within the extended Nambu-JonaLasinio model of Ref. [19].

The higher spin Regge recurrences arise thus from two kinds of composite spin- $J$ operators stemming from (1) and (2),

$$
\begin{aligned}
V_{\mu_{1} \mu_{2} \mu_{3} \ldots \mu_{J}} & =\bar{q} \gamma_{\mu_{1}} D_{\mu_{2}} D_{\mu_{3}} \ldots D_{\mu_{J}} q, \\
\tilde{V}_{\mu_{1} \mu_{2} \mu_{3} \ldots \mu_{J}} & =\bar{q} D_{\mu_{1}} D_{\mu_{2}} D_{\mu_{3}} \ldots D_{\mu_{J}} q .
\end{aligned}
$$

The operators (7) interpolate the spin- $J$ quark-antiquark states in the Lorentz representation $\left(\frac{J}{2}, \frac{J}{2}\right)$ and have the chiral transformation properties of usual vector current (1) (and for this reason emerge naturally in QCD analysis of deep inelastic scattering via OPE), while the chiral and Lorentz properties of (8) are different. Contracting $n$ times the Lorentz indices we get an interpolating operator for the $n$-th radial excitation of corresponding spin- $(J-2 n)$ meson.

For high enough canonical dimensions, several covariant derivatives in (7) and (8) can be replaced by insertion of gluon field strength $G_{\mu \nu}$, the corresponding operators will interpolate hybrid states. For instance, the operator $\bar{q} \gamma^{\mu} G_{\mu \nu} D^{\nu} q$ couples to a scalar hybrid with the chiral properties of vector current (1). One can of course construct purely gluonic operators which are chiral singlets. The leading twist- 2 operators of this sort have the structure

$\tilde{G}_{\mu_{1} \mu_{2} \mu_{3} \ldots \mu_{J}}=G_{\mu_{1}}^{\rho} D_{\mu_{2}} D_{\mu_{3}} \ldots D_{\mu_{J-1}} G_{\mu_{J} \rho}$.

In relating holographic predictions to observable states one should keep in mind that the spectrum of holographic models by itself does not know about the chiral and Lorentz structure of underlying QCD operators, only canonical dimension and spin are essential.

\section{The soft wall model}

For demonstration of our main idea we will use the simplest Abelian version of the SW model [9] defined by the 5D action

$S=c^{2} \int d^{4} x d z \sqrt{g} e^{-a z^{2}}\left(-\frac{1}{4} F_{M N} F^{M N}+\frac{1}{2} m_{5}^{2} V_{M} V^{M}\right)$,

where $g=\left|\operatorname{det} g_{M N}\right|, F_{M N}=\partial_{M} V_{N}-\partial_{N} V_{M}, M, N=$ $0,1,2,3,4, c$ is a normalization constant for the vector field $V_{M}$, and the background space represents the Poincaré patch of the $\mathrm{AdS}_{5}$ space with the metric

$g_{M N} d x^{M} d x^{N}=\frac{R^{2}}{z^{2}}\left(\eta_{\mu \nu} d x^{\mu} d x^{\nu}-d z^{2}\right), \quad z>0$.

Here $\eta_{\mu \nu}=\operatorname{diag}(1,-1,-1,-1), R$ denotes the radius of $\mathrm{AdS}_{5}$ space, and $z$ is the holographic coordinate which is usually interpreted as the inverse energy scale. At each fixed $z$ one has the metric of flat 4D Minkowski space. According to the standard prescriptions of AdS/CFT correspondence [5,6] the $5 \mathrm{D}$ mass $m_{5}$ is determined by the behavior of $p$-form fields near the UV boundary $z=0$,

$m_{5}^{2} R^{2}=(\Delta-p)(\Delta+p-4)$,

where $\Delta$ means the scaling dimension of $4 \mathrm{D}$ operator dual to the corresponding 5D field on the UV boundary. We consider the vector case, thus $p=1$ and $m_{5}^{2} R^{2}=(\Delta-1)(\Delta-3)$. The minimal value of dimension for vector operator in QCD is 
$\Delta=3$ (the current (1)) that, according to (12), corresponds to massless $5 \mathrm{D}$ vector fields which are usually considered in the SW models. But in general QCD operators interpolating vector mesons can have higher canonical dimensions, in particular, as was discussed in Sect. 2, the "descendants" preserving the chiral and Lorentz properties will have dimensions

$\Delta=3+2 k, \quad k=0,1,2, \ldots$

The 4D mass spectrum of KK modes can be found, as usual, from the equation of motion accepting the 4D planewave ansatz $V_{M}\left(x_{\mu}, z\right)=e^{i p x} v(z) \epsilon_{\mu}$ with the on-shell, $p^{2}=m^{2}$, and transverse, $p^{\mu} \epsilon_{\mu}=0$, conditions. In addition, we will imply the condition $V_{z}=0$ for the physical components of 5D fields [34]. For massless vector fields, this is equivalent to the standard choice of axial gauge due to emerging gauge invariance [9]. The ensuing from action (10) equation of motion is

$\partial_{z}\left(\frac{e^{-a z^{2}}}{z} \partial_{z} v_{n}\right)=\left(\frac{m_{5}^{2} R^{2}}{z^{3}}-\frac{m_{n}^{2}}{z}\right) e^{-a z^{2}} v_{n}$.

The particle-like excitations correspond to normalizable solutions of Sturm-Liouville equation (14). It is known that they form an infinite discrete set $v_{n}(z)$. The given property becomes more transparent after the substitution

$v_{n}=z^{1 / 2} e^{a z^{2} / 2} \psi_{n}$,

which transforms the Eq. (14) into a form of one-dimensional Schrödinger equation

$-\partial_{z}^{2} \psi_{n}+V(z) \psi_{n}=m_{n}^{2} \psi_{n}$,

with the potential

$V(z)=a^{2} z^{2}+\frac{1+m_{5}^{2} R^{2}-1 / 4}{z^{2}}$,

The mass spectrum of the model is given by the eigenvalues of Eq. (16),

$m_{n}^{2}=2|a|\left(2 n+1+\sqrt{1+m_{5}^{2} R^{2}}\right), \quad n=0,1,2, \ldots$

Using Eq. (12) for $p=1$ the spectrum can be rewritten as

$m_{n}^{2}=2|a|(2 n+\Delta-1)$.

The substitution of relation (13) into this spectrum leads to a remarkably simple prediction

$m_{n}^{2}=4|a|(n+k+1), \quad n, k=0,1,2, \ldots$

The formula (20) demonstrates that within the standard SW holographic model, the description of radial spectrum for vector mesons by interpolating HDO is essentially the same as by KK modes - the numbers $n$ and $k$ can be interchanged.

The spectral relation (20) can be now used for relating the KK modes to excited mesons in QCD. The lightest KK mode, $n=0$, corresponds to a vector state interpolated by the usual vector current (1) of canonical dimension $\Delta=3$, the standard case of $\rho(770)$ and $\omega(782)$ mesons. The mass of $n=1 \mathrm{KK}$ mode of field dual to the operator (1) coincides with the mass of $n=0$ mode of field dual to the operator (4) with canonical dimension $\Delta=5$. We identify them and interpret the given state as coupled to two interpolating operators. This can be continued to arbitrary $n$-th radial excitation - it will be coupled to a set of $n+1$ interpolating operators with growing canonical dimensions. Thus the physical interpretation and description of radially excited states become close in spirit to the description from first principles in lattice simulations [11].

It is not difficult to show that the same property and interpretation holds for other integer spins. First let us consider the action for a free scalar field $\Phi$ on $\mathrm{AdS}_{5}$ with the dilaton background as in (10),

$S=\frac{1}{2} c^{2} \int d^{4} x d z \sqrt{g} e^{-a z^{2}}\left(\partial_{M} \Phi \partial^{M} \Phi-m_{5}^{2} \Phi^{2}\right)$.

As before, the physical 4D modes are given by the planewave ansatz $\Phi\left(x_{\mu}, z\right)=e^{i p x} \phi(z)$. The equation of motion reads,

$\partial_{z}\left(\frac{e^{-a z^{2}}}{z^{3}} \partial_{z} \phi_{n}\right)=\left(\frac{m_{5}^{2} R^{2}}{z^{5}}-\frac{m_{n}^{2}}{z^{3}}\right) e^{-a z^{2}} \phi_{n}$,

which after the substitution $\phi_{n}=z^{3 / 2} e^{a z^{2} / 2} \psi_{n}$ transforms into the Schrödinger Eq. (16) with the potential

$V(z)=a^{2} z^{2}+\frac{4+m_{5}^{2} R^{2}-1 / 4}{z^{2}}+2 a$.

The corresponding eigenvalues are

$m_{n}^{2}=2|a|\left(2 n+1+\frac{a}{|a|}+\sqrt{4+m_{5}^{2} R^{2}}\right), \quad n=0,1,2, \ldots$

Again making use of Eq. (12) but for $p=0$ in (24) we obtain

$m_{n}^{2}=2|a|\left(2 n+\frac{a}{|a|}+\Delta-1\right)$.

Since the simplest scalar QCD operator shown in (2) has $\Delta=$ 3 , the relation (13) for dimensions of scalar "descendants" can be applied, the radial scalar spectrum takes the form,

$m_{n}^{2}=4|a|\left(n+k+1+\frac{a}{2|a|}\right), \quad n, k=0,1,2, \ldots$,

which differs from the vector spectrum (20) only by a general shift depending on the sign of constant $a$ in the dilaton background.

Now we will generalize the model to the case of arbitrary spin. The higher spin fields $\Phi_{J} \doteq \Phi_{M_{1} M_{2} \ldots M_{J}}, M_{i}=$ $0,1,2,3,4$, are described by symmetric, traceless tensors of 
rank $J$. By assumption, the physical components of 5D fields satisfy the condition,

$\Phi_{z \ldots}=0$,

which is a generalization of condition $V_{z}=0$ used in the vector case. The constraint $\partial^{\mu} \Phi_{\mu \ldots}=0$ is also imposed to have the required $2 J+1$ physical degrees of freedom on the $4 \mathrm{D}$ boundary. In general, an action for free higher spin fields contains many quadratic terms appearing from many ways of contraction of coordinate indices. But the condition (27) greatly simplifies the action leaving two terms only $[9,34]$,

$I=(-1)^{J} \frac{1}{2} \int d^{4} x d z \sqrt{g} e^{-a z^{2}}\left(\nabla_{N} \Phi_{J} \nabla^{N} \Phi^{J}-m_{J}^{2} \Phi_{J} \Phi^{J}\right)$.

It is known that the covariant derivatives $\nabla$ in the SW action (28) can be effectively replaced by the usual ones if the mass $m_{J}^{2}$ is shifted, $m_{J}^{2} \rightarrow b_{J} z^{2}+c_{J}$, where $a_{J}$ and $b_{J}$ are certain $J$-dependent constants emerging from affine connection in $\nabla$ [34]. They will entail a certain $J$-dependent contribution (a general shift) to the spectrum of normalizable modes. The final effect of these terms turns out to be the same as if we used in (28) just normal derivatives and the five-dimensional mass were $[34,35]$

$m_{J}^{2} R^{2}=(\Delta-J)(\Delta+J-4)$.

The $J$-dependent mass (29) is nothing but the relation (12) with " $p$ " replaced by " $J$ ". As was demonstrated in Ref. [36, 37], the origin of relation (29) lies in the the condition (27) which leads to decoupling of kinematical aspects from dynamical ones with final outcome that symmetric tensors of rank $p$ (describing mesons of spin $p$ ) obey the same equation as the $p$-form fields.

We will exploit all these findings to simplify the treatment of tensor mesons: The covariant derivatives $\nabla$ in the action (28) will be replaced by the normal ones with simultaneous use of the relation (29). Imposing again the 4D planewave ansatz $\Phi_{J}\left(x_{\mu}, z\right)=e^{i p x} \phi^{(J)}(z) \epsilon_{J}$, where $\epsilon_{J}$ denotes polarization, we get the equation of motion for the profile function $\phi^{(J)}(z)$ of physical 4D modes,

$$
\begin{aligned}
& -\partial_{z}\left(e^{-a z^{2}} z^{2 J-3} \partial_{z} \phi_{n}^{(J)}\right)+m_{J}^{2} R^{2} e^{-a z^{2}} z^{2 J-5} \phi_{n}^{(J)} \\
& =m_{n}^{2} e^{-a z^{2}} z^{2 J-3} \phi_{n}^{(J)} .
\end{aligned}
$$

The substitution $\phi_{n}^{(J)}=e^{a z^{2} / 2} z^{3 / 2-J} \psi_{n}^{(J)}$ casts the Eq. (30) into the Schrödinger Eq. (16) with the potential

$V(z)=a^{2} z^{2}+\frac{(J-2)^{2}+m_{J}^{2} R^{2}-1 / 4}{z^{2}}+2 a(1-J)$

The discrete mass spectrum is

$$
m_{n, J}^{2}=2|a|\left(2 n+1+\frac{a}{|a|}(1-J)+\sqrt{(J-2)^{2}+m_{J}^{2} R^{2}}\right) .
$$

Substituting relation (29), we obtain the final result

$m_{n, J}^{2}=2|a|\left(2 n+\frac{a}{|a|}(1-J)+\Delta-1\right)$.

It is easy to see that all corresponding equations for vector and scalar mesons considered above represent just particular cases $J=1$ and $J=0$ of presented relations for arbitrary integer spin. Now we need the last ingredient - an extension of expression for canonical dimension of interpolating operators (13) to the case of arbitrary spin. As follows from the discussions in Sect. 2, this extension of (13) is

$\Delta=2+J+2 k, \quad k=0,1,2, \ldots$,

which holds for $J \geq 1$. From the Eq. (33) we then obtain for non-zero spins,

$m_{n, J}^{2}=4|a|\left(n+k+\frac{1}{2}(1+J)+\frac{a}{2|a|}(1-J)\right)$.

For consistency, we must choose the sign $a<0$ in the dilaton background because for $a>0$ the spectrum does not depend on spin, $m_{n, J}^{2}=4 a(n+k+1)$. Thus, the final relation is

$m_{n, J}^{2}=4|a|(n+k+J), \quad n, k=0,1,2, \ldots, \quad J>0$.

The relation (36) generalizes the spectrum of the simplest SW model [9] for the twist-two interpolating operators $(k=0)$ to higher twists. The choice of sign for $a$ is in fact predetermined by the way one introduces the higher spin fields. If we introduced them following the original paper on SW model [9] as gauge massless fields on $\mathrm{AdS}_{5}$, the situation would be opposite - the mass spectrum (36) would be obtained for $a>0$ (this happens due to a specific rescaling of fields, see discussions on this point in Ref. [38]). But independently of the issue of sign, we come to a remarkable conclusion that the integer number $n$ in (36) enumerating the KK excitation can be replaced by the integer number $k$ enumerating the higher-dimensional interpolating operators with identical chiral and Lorentz properties which can be built in QCD. Thus we obtain a new physical interpretation for this number that historically gave rise to the notion of daughter Regge trajectories in old Veneziano dual amplitudes and in QCD string models.

It is important to emphasize that the structure of the Regge trajectories remains encoded in the geometry but in indirect way - via the boundary behavior of fields that dictates the $J$-dependence of 5D masses in the relation (29).

We can get a further insight from consideration of normalized eigenfunctions corresponding to the discrete spectrum (36),

$\phi_{n}^{(J)}=\sqrt{\frac{2 n !}{(J+2 k+n) !}} e^{-|a| z^{2}}\left(|a| z^{2}\right)^{1+k} L_{n}^{J+2 k}\left(|a| z^{2}\right)$, 
where $L_{n}^{\alpha}(x)$ are associated Laguerre polynomials. It is seen that the numbers $n$ and $k$ are not completely interchangeable in the radial wave function: While the large $z$ asymptotics depends on the sum $n+k$ (because $L_{n}^{\alpha}(x) \sim x^{n}$ at large $x$ ), the number of zeros is controlled by $n$ only (as the polynomial $L_{n}^{\alpha}(x)$ has $n$ zeros). By setting $n=0$, i.e. by keeping the zero KK mode only, we thus choose the wave function without zeros in holographic coordinate. This wave function is the least "entangled" with the 5th holographic dimension and thereby is the least sensitive to deviations from the AdS structure. As we will discuss in Sect. 5, this makes the zero KK mode the most reliable in the phenomenological holographic approaches.

The real QCD operators have anomalous dimensions and this represents a notorious problem for the whole bottom-up holographic approach. One makes reference to asymptotic freedom at best, any serious discussion of this problem is usually avoided. We will not give a real physical justification but make an observation. Within our considerations, the account for the anomalous dimension of operators is tantamount to replacement $k \rightarrow k+\varepsilon(k, J)$ in the spectrum (36). Then $2 \varepsilon(k, J)$ (see Eq. (13)) reflects contribution to the canonical dimension $\Delta$ from the anomalous part. The systematic form of $\varepsilon(k, J)$ is unknown but it is naturally expected that $\varepsilon(k, J)$ is a growing function of both arguments. However, the spectrum (36) more or less meets the existing phenomenology [23,28-33,40]. This should mean that $\varepsilon(k, J)$ is either suppressed in the large- $N_{c}$ limit (perhaps the size of $\varepsilon(k, J)$ can be then systematically estimated by a phenomenological analysis of deviations from the relation (36)) or by itself is an approximate linear function of its arguments (hence, the effects of anomalous dimensions are then effectively absorbed by the phenomenological values of parameters in (36)). The both possibilities could constitute an interesting prediction of the SW holographic approach.

\section{The Hard Wall model}

The spectrum of HW holographic model $[7,8]$ is not Regge like, on the other hand, this model is much better accommodated for description of the chiral symmetry breaking in QCD and hadron form factors. The analysis of previous section can be easily applied to the HW model - one can just set $a=0$ in the corresponding equations and impose the infrared cutoff $z_{m}$. The normalizable solution of Eq. (30) satisfying $\phi(0)=0$ is then given by

$\phi \sim z^{2-J} J_{\Delta-2}(m z)$,

where $J_{\alpha}(x)$ is the Bessel function. The solution (38) corresponds to interpolating QCD operator of canonical dimension $\Delta$. In the original papers $[7,8]$, the discrete spectrum emerges from the Dirichlet boundary condition,

$\partial_{z} \phi\left(m_{n} z_{m}\right)=0$.

We find more convenient to introduce the higher spin fields into the HW model in the gauge invariant way - the way adopted in the original SW model [9]. Actually this analysis in the HW model was carried out for fields dual to twist-2 operators in Ref. [39], we can directly take the final result and generalize it to arbitrary twists. In brief, the ensuing equation of motion is tantamount to making the substitution $\phi^{(J)}=z^{4-J-\Delta} \tilde{\phi}^{(J)}$ (the field $\tilde{\phi}^{(J)}$ becomes constant at the UV boundary $z \rightarrow 0$ ) and dropping the 5D mass term in Eq. (30) with $a=0$. We get

$-\partial_{z}\left(z^{1-2(\Delta-2)} \partial_{z} \tilde{\phi}_{n}^{(J)}\right)=m_{n}^{2} z^{1-2(\Delta-2)} \tilde{\phi}_{n}^{(J)}$.

The normalizable solution satisfying $\tilde{\phi}^{(J)}(0)=0$ is

$\tilde{\phi}^{(J)} \sim z^{\Delta-2} J_{\Delta-2}(m z)$.

The Dirichlet boundary condition leads to the following equation for discrete spectrum,

$J_{\Delta-3}\left(m_{n} z_{m}\right)=0$,

where the property of Bessel functions $\partial_{x}\left(x^{\alpha} J_{\alpha}\right)=x^{\alpha} J_{\alpha-1}$ was exploited. Setting $\Delta=J+2$ in Eq. (42), we obtain the equation of Ref. [39] for spectrum of higher spin mesons interpolated by twist- 2 operators.

Substituting to the Eq. (42) different canonical dimensions $\Delta$ we will get radial spectra which do not coincide in any part because zeros of different Bessel functions have different set of locations. This shows that the KK modes of HW model can not be traded for states arising from HDO.

It is interesting to notice, however, that if we consider the spectrum of $n=0$ modes for $\Delta=3,4,5, \ldots$ in the Eq. (42), we get the sequence of roots: $m_{0}(\Delta) z_{m} \approx$ $\{2.4,3.8,5.1,6.4,7.6, \ldots\}$. For the usual inputs $z_{m}^{-1} \approx$ $323 \mathrm{MeV}$ and $m_{0}(\Delta=3)=776 \mathrm{MeV}$ of the HW model [7], these roots lead to the mass spectrum (in $\mathrm{MeV}$ )

$m_{0}(\Delta) \approx\{776,1234,1653,2056,2452, \ldots\}$.

The given spectrum yields much slower rise of masses in the radial trajectories than in the traditional HW approach (namely $m_{\rho, n} \approx\{776,1777,2810,3811, \ldots\} \mathrm{MeV}$ in Ref. [7]). This provides a qualitatively better spectroscopy: In the mass range below $2.5 \mathrm{GeV}$ (relatively well scanned experimentally for light non-strange mesons) we obtain 5 $\rho$-mesons that is more or less in accord with the Particle Data [24], while the standard HW model predicts only $2 \rho$ mesons. It is also curious to observe that the spectrum (43) interpolates with a good precision the experimental positions for clusters of light non-strange meson resonances arising from approximate mass degeneracies between radial and spin (or orbital) excitations [23,28-33,40]. This may be viewed 
as a phenomenological indication that in the HW model, only zero $K K$ modes of fields dual to HDO in QCD have physical significance.

\section{Discussions}

The KK modes are usually regarded as inherent for holographic models and are widely used in phenomenological applications. As we mentioned in Introduction, their physical properties, however, are very different from those of hadron resonances [10]. Below we draw attention to the fact that the KK modes and resonances in confining theory, in a sense, come from different sectors from the point of view of original AdS/CFT correspondence [3,4].

In the compactified AdS space of HW models, the nature of $\mathrm{KK}$ modes is similar to $\mathrm{KK}$ modes from flat extra space compactified on a circle - they emerge from boundary conditions on propagation of particles in the whole extra dimension and the higher is a KK-mode, the stronger is "entangled" its wave function with extra dimension (has more zeros). In the infinite AdS space - the case of SW model - the nature of KK modes becomes more tricky. The gravitational potential grows in spaces with negative lambda-term, as a result the gravity acts on massive particles as a kind of potential well [41]. Massless particles in AdS are known to reach the boundary at infinity for finite time [41], so they can be also considered as living in a gravitational box. As before, the KK modes are well-defined if the whole infinite AdS space is available for propagation. The problem of reliability of KK modes in practical holographic models arises from the fact that the $\mathrm{AdS}_{5}$ space in the AdS/CFT correspondence is an approximation which is valid only in the deep infrared domain.

To clarify the point, it should be recalled that the Maldacena's gauge/gravity conjecture originated from the threebrane supergravity solution for a stack of $N_{c}$ coincident 3D branes in 10D ambient space [3,4]. The corresponding extremal solution for the sign convention as in (11) has the form (omitting the 4-form RR-potential)

$$
\begin{aligned}
d s^{2} & =\left(1+\frac{R^{4}}{r^{4}}\right)^{-\frac{1}{2}} d y_{\mu}^{2}-\left(1+\frac{R^{4}}{r^{4}}\right)^{\frac{1}{2}}\left(d r^{2}+r^{2} d \Omega_{5}^{2}\right), \\
r^{2} & =\sum_{i=4}^{9} y_{i}^{2}, \quad R^{4}=4 \pi g_{s} N_{c} l_{s}^{4}=g_{\mathrm{YM}}^{2} N_{c} l_{s}^{4}=\lambda l_{s}^{4},(44)
\end{aligned}
$$

where the indices $\mu=0,1,2,3$ run along the worldvolume of D3 branes, $\Omega_{5}$ denotes unit 5-sphere, $l_{s}$ and $g_{s}$ are the string length and coupling. The gravitational description is justified for $R \gg l_{s}$ that entails the large 't Hooft coupling $\lambda \gg 1$. The conjecture of AdS/CFT correspondence emerges in the region $r \ll R$, where the metric (44) factorizes into
$\operatorname{AdS}_{5} \times \mathrm{S}_{5}$,

$$
\left.d s^{2}\right|_{r \ll R}=\frac{r^{2}}{R^{2}} d y_{\mu}^{2}-\frac{R^{2}}{r^{2}} d r^{2}-R^{2} d \Omega_{5}^{2} .
$$

Strictly speaking, one obtains in (45) the Poincaré patch of $\mathrm{AdS}_{5}$ describing only half of the global $\mathrm{AdS}_{5}$ space (a region $r>0$ lying from "one side" of the $\mathrm{D} 3$ stack). After the change of coordinate $r=R^{2} / z$ this patch takes the form of (11). We see thus that the metric (45) in holographic QCD has a justification within the original gauge/gravity duality for $z \gg R$ only. The matching to QCD, however, is usually performed in the limit $z \rightarrow 0$ where the metric (44) does not have an AdS part at all. Since the original AdS/CFT correspondence does not have matter in the fundamental representation, a better example could be the top-down holographic approach [42] - after geometrical introduction of flavor branes and of chiral symmetry breaking, the metric of resulting low energy models has nothing in common with AdS.

These remarks may suggest that the higher KK modes lie outside the applicability of the bottom-up holographic approach. On the other hand, they are indispensable for internal consistency as they provide the correct high energy behavior of correlation functions. According to the analysis of our work, these higher KK modes can be effectively replaced by infinite number of zero KK modes stemming from heavier fields dual to HDO in QCD. This removes the aforementioned discrepancy. Perhaps one can develop a more solid justification by imposing the Pauli-Villars regularization in line with the compactified $\mathrm{AdS}_{5}$ (i.e. Randall-Sundrum) extradimensional scenario of Ref. [43] - this regularization effectively suppresses the propagation of KK modes of gauge fields except zero mode.

The linear form of radial spectrum of SW model in our scheme remains unchanged and the proposed approach by itself cannot explain the origin of quadratic dilaton background in the action (10). This long-standing problem is still open. The original proposal for the dilaton factor $e^{\varphi}$ was inspired by the string theory but the quadratic form, $\varphi=-a z^{2}$, speculatively conceivable as a possible result of "closed string tachyon condensation" [9], was completely phenomenological. A gravitational theory with quadratic dilaton background is not known. There are numerous attempts in the literature to develop a sort of dynamical Maxwell-Dilaton-gravity model, however, the desired background $e^{-a z^{2}}$ on $\mathrm{AdS}_{5}$ space either does not appear even approximately or is obtained at the cost of introduction of some complicated potential $V(\varphi)$ in the action. Without any theoretical restrictions on the form of $V(\varphi)$ (e.g., like renormalizability) such a solution looks as $a d$ hoc as introduction of this background by hands. Some relevant discussions of this problem are given in Ref. [44]

It is not excluded that a viable solution lies in a completely different direction. For instance, one may speculate 
that this background can be interpreted as an effective way for taking into account some quantum features of particle propagation in the AdS space-time, in which gravity effectively acts as a finite box. In this respect, it is interesting to mention the recent work [45] where it was demonstrated that in Lorentzian AdS, gravity dresses free propagators on the quantum level. In particular, the scalar component of 5D graviton leads to universal exponential suppression of propagators in the infrared region,

$\Delta_{p}(z) \propto e^{-\alpha p z}$,

where $p=\sqrt{p^{\mu} p_{\mu}}$ and positive constant $\alpha \sim 1 /\left(R M_{\mathrm{Pl}}\right)^{2}$ arises from one-loop gravitational corrections. The one-loop corrections due to interactions with other fields in the bulk (including self-interaction) also lead to the suppression of the kind (46) [45]. In other words, the AdS space turns out to be opaque to propagation in deep infrared region. Basing on this result, we may imagine the following. At high enough energy one has $p \sim E_{\mathrm{p}}$, where $E_{\mathrm{p}}$ is the particle proper energy (the energy measured in the reference frame instantaneously at rest at $z$ ). The proper energy is related to the energy in the gauge theory $E_{\text {exp }}$, that is measurable experimentally, via the rescaling dictated by the AdS metric (11),

$p \sim E_{\mathrm{p}}=\frac{z}{R} E_{\mathrm{exp}}$.

Then if we want to deal with measurable quantities (as we do in the bottom-up holographic QCD) we should substitute (47) into (46),

$\Delta_{p}(z) \propto e^{-|a| z^{2}}$,

where $a$ is a constant proportional to a typical energy scale multiplied by $\mathrm{AdS}_{5}$ curvature $1 / R$ and by $5 \mathrm{D}$ gravity coupling squared from one-loop gravitational corrections. Loosely speaking, the $\operatorname{dim} 2$ slope parameter $a$ of meson trajectories emerges as a result of multiplication of two dim1 factors stemming from the UV and IR dynamics correspondingly. A similar interpretation for $a$ was proposed in Refs. [46,47] from different considerations.

The asymptotic behavior (48) can be compared with the bulk-to-boundary propagators in the SW model. In the vector case (10) (see, e.g., Ref. [48]),

$\Delta_{p}(z)=\Gamma\left(1-\frac{p^{2}}{4|a|}\right) e^{(a-|a|) z^{2} / 2} U\left(\frac{-p^{2}}{4|a|}, 0 ;|a| z^{2}\right)$,

where $U(\alpha, \beta ; x)$ is the Tricomi confluent hypergeometric function which vanishes for $x \rightarrow \infty$ as $x^{-\alpha}$. For arbitrary spin $\Delta_{p}(z)$ is qualitatively similar - the main change is $\beta=|J-1|$ in $U(\alpha, \beta ; x)$ [34]. Now one can observe that at $a<0$ in (49) - the choice that we made in Sect. 3 for arbitrary spin - the propagators in the SW model acquire the same exponential suppression as in (48). The dilaton background of the SW model might be thus viewed as an effective account for corrections to particle propagators in the $\mathrm{AdS}_{5}$ space stemming from quantum gravity and other possible interactions in the bulk.

\section{Conclusions}

In the usual AdS/QCD approach, the radially excited hadrons are identified with Kaluza-Klein excitations of dual theory. This has, however, severe theoretical problems. We proposed an alternative description in which the excited modes become a superposition of several Kaluza-Klein states arising from massive $5 \mathrm{D}$ fields dual to $\mathrm{QCD}$ operators with different canonical dimensions. Exactly along this line the radially excited states are extracted in the lattice simulations from a set of interpolating QCD operators. The new description was applied to the Soft Wall and Hard Wall holographic models in the sector of light mesons. The linear radial and angular Regge spectrum of Soft Wall model remains unchanged. The radial spectrum of Hard Wall model changes significantly. We consider this result as another strong indication (in addition to non-Regge behavior) on the fact that the KK modes of HW model can not be identified with real mesons in the large- $N_{c}$ limit of QCD.

The approach proposed in the present work may be interpreted as a simple tool establishing a direct connection between composite QCD operators and hadron resonances with a definite mass. The emerging connection becomes thus a new nice feature of bottom-up holographic models. Contrary to many theoretical approaches aimed at description of excited hadrons, the description in AdS/QCD becomes close in spirit to the methods used in lattice simulations [11].

As the presented holographic approach to hadron spectroscopy is more tightly related with the real QCD than the traditional bottom-up holographic models, it would be interesting to explore it beyond the hadron spectroscopy. We leave this work for future.

Data Availability Statement This manuscript has no associated data or the data will not be deposited. [Authors' comment: There are no external data associated with the manuscript.]

Open Access This article is licensed under a Creative Commons Attribution 4.0 International License, which permits use, sharing, adaptation, distribution and reproduction in any medium or format, as long as you give appropriate credit to the original author(s) and the source, provide a link to the Creative Commons licence, and indicate if changes were made. The images or other third party material in this article are included in the article's Creative Commons licence, unless indicated otherwise in a credit line to the material. If material is not included in the article's Creative Commons licence and your intended use is not permitted by statutory regulation or exceeds the permitted use, you will need to obtain permission directly from the copyright holder. To view a copy of this licence, visit http://creativecomm 
ons.org/licenses/by/4.0/.

Funded by $\mathrm{SCOAP}^{3}$.

\section{References}

1. G. 't Hooft, Nucl. Phys. B 72, 461 (1974)

2. E. Witten, Nucl. Phys. B 160, 57 (1979)

3. J.M. Maldacena, Adv. Theor. Math. Phys. 2, 231 (1998)

4. J.M. Maldacena, Int. J. Theor. Phys. 38, 1113 (1999)

5. E. Witten, Adv. Theor. Math. Phys. 2, 253 (1998)

6. S.S. Gubser, I.R. Klebanov, A.M. Polyakov, Phys. Lett. B 428, 105 (1998)

7. J. Erlich, E. Katz, D.T. Son, M.A. Stephanov, Phys. Rev. Lett. 95, $261602(2005)$

8. L. Da Rold, A. Pomarol, Nucl. Phys. B 721, 79 (2005)

9. A. Karch, E. Katz, D.T. Son, M.A. Stephanov, Phys. Rev. D 74, 015005 (2006)

10. C. Csaki, M. Reece, J. Terning, JHEP 0905, 067 (2009)

11. J.J. Dudek et al., Phys. Rev. D 82, 034508 (2010)

12. G. Colangelo, F. Hagelstein, M. Hoferichter, L. Laub, P. Stoffer, JHEP 03, 101 (2020)

13. J. Leutgeb, A. Rebhan, Phys. Rev. D 101, 114015 (2020)

14. L. Cappiello, O. Cata, G. D’Ambrosio, D. Greynat, A. Iyer, arXiv:1912.02779 [hep-ph]

15. A.A. Andrianov, V.A. Andrianov, Int. J. Mod. Phys. A 8, 1981 (1993)

16. A.A. Andrianov, V.A. Andrianov, V.L. Yudichev, Theor. Math. Phys. 108, 1069 (1996) [Teor. Mat. Fiz. 108, 276 (1996)]

17. V.A. Andrianov, S.S. Afonin, Eur. Phys. J. A 17, 111 (2003)

18. V.A. Andrianov, S.S. Afonin, Zap. Nauchn. Semin. 291, 5 (2002). arXiv:hep-ph/0304140

19. M.V. Chizhov, arXiv:hep-ph/9610220

20. T.D. Cohen, X.D. Ji, Phys. Rev. D 55, 6870 (1997)

21. M. Shifman, arXiv:hep-ph/0507246

22. L.Y. Glozman, Phys. Rept. 444, 1 (2007)

23. M. Shifman, A. Vainshtein, Phys. Rev. D 77, 034002 (2008)
24. M. Tanabashi et al. (Particle Data Group), Phys. Rev. D 98, 030001 (2018)

25. M.A. Shifman, A.I. Vainstein, V.I. Zakharov, Nucl. Phys. B 147 385, 448 (1979)

26. L.J. Reinders, H. Rubinstein, S. Yazaki, Phys. Rept. 127, 1 (1985)

27. P. Colangelo, A. Khodjamirian, arXiv:hep-ph/0010175

28. S.S. Afonin, Eur. Phys. J. A 29, 327 (2006)

29. S.S. Afonin, Phys. Lett. B 639, 258 (2006)

30. S.S. Afonin, Phys. Rev. C 76, 015202 (2007)

31. S.S. Afonin, Mod. Phys. Lett. A 22, 1359 (2007)

32. S.S. Afonin, Int. J. Mod. Phys. A 22, 4537 (2007)

33. E. Klempt, A. Zaitsev, Phys. Rept. 454, 1 (2007)

34. S.J. Brodsky, G.F. de Teramond, H.G. Dosch, J. Erlich, Phys. Rept. 584, 1 (2015)

35. T. Gutsche, V.E. Lyubovitskij, I. Schmidt, A. Vega, Phys. Rev. D 85, 076003 (2012)

36. G.F. de Teramond, S.J. Brodsky, Phys. Rev. Lett. 102, 081601 (2009)

37. G.F. de Teramond, H.G. Dosch, S.J. Brodsky, Phys. Rev. D 87, 075005 (2013)

38. S.S. Afonin, Adv. High Energy Phys. 2017, 8358473 (2017)

39. E. Katz, A. Lewandowski, M.D. Schwartz, Phys. Rev. D 74, 086004 (2006)

40. D.V. Bugg, Phys. Rept. 397, 257 (2004)

41. B. Zwiebach, A First Course in String Theory, 2nd edn. (Cambridge University Press, Cambridge)

42. J. Erdmenger, N. Evans, I. Kirsch, Threlfall, Eur. Phys. J. A 35, 81 (2008)

43. A. Pomarol, Phys. Rev. Lett. 85, 4004 (2000)

44. J. Sonnenschein, Prog. Part. Nucl. Phys. 92, 1 (2017)

45. S. Fichet, Phys. Rev. D 100, 095002 (2019)

46. S.S. Afonin, Phys. Lett. B 675, 54 (2009)

47. S.S. Afonin, Phys. Lett. B 678, 477 (2009)

48. S.S. Afonin, Int. J. Mod. Phys. A 27, 1250171 (2012) 